\title{
Quantitative Antibiogram as a Typing Method FOR THE PROSPECTIVE EPIDEMIOLOGICAL SURVEILlaNCE AND CONTROL OF MRSA: COMPARISON WiTH MOLECULAR TyPING
}

\author{
Dominique S. Blanc, PhD; Christiane Petignat, MD; Philippe Moreillon, MD, PhD; \\ Aline Wenger, MSc; Jacques Bille, MD; Patrick Francioli, MD
}

See also pages 649-653 and pages 660-667.

\begin{abstract}
OBJECTIVE: Evaluation of the quantitative antibiogram as an epidemiological tool for the prospective typing of methicillin-resistant Staphylococcus aureus (MRSA), and comparison with ribotyping.

METHODS: The method is based on the multivariate analysis of inhibition zone diameters of antibiotics in disk diffusion tests. Five antibiotics were used (erythromycin, clindamycin, cotrimoxazole, gentamicin, and ciprofloxacin). Ribotyping was performed using seven restriction enzymes (EcoRV, HindIII, KpnI, PstI, EcoRI, SfuI, and Bam HI).

SETTING: 1,000-bed tertiary university medical center.

RESULTS: During a 1-year period, 31 patients were found to be infected or colonized with MRSA. Cluster analysis of antibiogram data showed nine distinct antibiotypes. Four antibiotypes were isolated from multiple patients $(2,4$,

7 , and 13 , respectively). Five additional antibiotypes were isolated from the remaining five patients. When analyzed with respect to the epidemiological data, the method was found to be equivalent to ribotyping.

Among 206 staff members who were screened, six were carriers of MRSA. Both typing methods identified concordant of MRSA types in staff members and in the patients under their care.

CONCLUSIONS: The quantitative antibiogram was found to be equivalent to ribotyping as an epidemiological tool for typing of MRSA in our setting. Thus, this simple, rapid, and readily available method appears to be suitable for the prospective surveillance and control of MRSA for hospitals that do not have molecular typing facilities and in which MRSA isolates are not uniformly resistant or susceptible to the antibiotics tested (Infect Control Hosp Epidemiol 1996;17:654-659).
\end{abstract}

\section{INTRODUCTION}

Accurate typing methods are very important for optimal infection control. With the advance of molecular technology, typing methods with high versatility, typeability, reproducibility, and discrimination have been developed. However, these methods probably will never be available in many hospitals because of the complex technology and costs necessary for their implementation.

Patterns of susceptibility to antimicrobial agents have been used widely for typing purposes, because they are readily available, easy to determine, and relatively inexpensive. However, an inherent weakness of this method is that changes in antimicrobial susceptibility phenotypes often are related to environmental factors or plasmids. Furthermore, the method is not always reproducible when the same isolate is tested several times, and it has been shown that methicillin-resistant Staphylococcus aureus (MRSA) isolates that were indistinguishable by antibiotic susceptibility tests could be discriminated on the basis of their genotypes. ${ }^{1-6}$

In an attempt to enhance the value of the antibi-

From the Division of Hospital Preventive Medicine (Drs. Blanc, Petignat, and Francioli), the Division of Infectious Diseases (Dr. Moreillon), and the Institute of Microbiology (Drs. Bille and Wenger), Centre Hospitalier Universitaire Vaudois, Lausanne, Switzerland.

Presented in part at the third International Meeting on Bacterial Epidemiological Markers, April 1994, Cambridge, United Kingdom (poster PA1).

The authors are grateful to D. Raffalli and to I. Vernez for technical assistance.

Address reprint requests to Dominique Blanc, Division automnome de médecine préventive hospitalière, Centre Hospitalier Univertitaire Vaudois, CH-1011 Lausanne, Switzerland.

95-OA-009. Blanc DS, Petignat C, Moreillon P, Wenger A, Bille J, Francioli P. Quantitative antibiogram as a typing method for the prospective epidemiological surveillance and control of MRSA: comparison with molecular typing. Infect Control Hosp Epidemiol 1996;17:654-659. 
ogram as a tool for nosocomial infection surveillance, a similarity analysis of susceptibility testing results that takes into consideration the diameters of growth inhibition zones in disk diffusion tests has been proposed and found promising for the analysis of MRSA. ${ }^{7,8}$ In a retrospective evaluation, we compared this method with ribotyping for the analysis of MRSA in our hospital and found it suitable for epidemiological purposes. ${ }^{8}$ In the present study, this method was evaluated for the prospective surveillance and control of MRSA.

\section{MATERIAL AND METHODS Epidemiological Setting and Infection Control Strategies}

The university hospital of Lausanne is a 1,000bed tertiary-care teaching hospital. The hospital has 40 intensive-care beds and a total of approximately 30,000 admissions per year. It serves a population of approximately 300,000. Frequency of methicillin resistance among $S$ aureus isolates (one isolate per patient) varied between $1.8 \%$ and $3.9 \%$ during the last 5 years. ${ }^{9}$

Patients with MRSA were identified by daily surveillance of microbiological laboratory data and by surveillance cultures of readmitted patients known to have been positive for MRSA. As soon as a patient was identified as colonized or infected with MRSA, he or she was placed on strict isolation, and daily control measures were implemented until eradication of MRSA or discharge of the patient. Details have been described elsewhere. ${ }^{9}$

As soon as a MRSA strain was isolated from a patient, antibiogram typing was done, and the similarity between this isolate and previous isolates of MRSA was computed. When several patients in the same ward were colonized or infected with isolates of similar antibiogram, cultures of anterior nares were performed of the hospital staff and all patients of the ward who had potential for contact with patients harboring MRSA.

\section{Ribotyping}

Ribotyping was performed as previously described $8,9,11$ To increase the discrimination between isolates, ribotyping of all isolates was performed using four additional restriction enzymes (PstI, EcoRI, SfuI, and BamHI) in addition to the three restriction enzymes previously used (EcoRV, HindIII, and $K p n \mathrm{I}) .8,9$

\section{Antibiogram Typing}

Antibiograms were performed by the conventional Kirby-Bauer disk diffusion test on MuellerHinton agar with 24 hours of incubation at $35^{\circ} \mathrm{C} .10$
The McFarland 0.5 inoculum was obtained using the Cobas Inocheck (Roche Diagnostic, Basel, Switzerland). Five antibiotics were used for typing purposes: erythromycin, clindamycin, cotrimoxazole, gentamicin, and ciprofloxacin. The criteria for the choice of the antibiotics were (1) variable and independent resistance among MRSA isolates, or (2) chromosomal resistance, or (3) relatively low frequency of use in the hospital. Diameters of growth inhibition were measured by a caliper.

Similarity analysis between isolates was performed using the Euclidean distance as a similarity coefficient, given by the following formula:

$$
E_{j k}=\sqrt{\sum_{i=1}^{n}\left(x_{i j}-x_{i k}\right)}
$$

where $x_{i j}$ and $x_{i k}$ are the inhibition diameter values of a given antibiotic $i$ for two distinct isolates $j$ and $k$. The greater the distance between two isolates, the smaller is the resemblance between them. Cluster analysis then was performed on similarity data using the unweighted pair groups method of analysis (UPGMA). Similarity analysis and cluster analysis were computed by the Numerical Taxonomy and Multivariate Analysis System (NTSYS-pc, version 1.70, Exeter Software, Setauket, NY).

\section{RESULTS \\ Ribotyping}

In 1993, 31 patients and 6 members of the hospital staff were found to be infected or colonized with MRSA. Nine different strains could be distinguished by ribotyping (Figure 1). Of these, four strains were isolated from 2 (cluster 4), 4 (cluster 2), 7 (cluster 3), and 13 patients (cluster 1), respectively. The remaining five strains were isolated from one patient each. All staff members were colonized with the same strain harbored by the patient for which they were responsible (see below).

The four supplementary restriction enzymes did not provide further discrimination, except for one isolate, which had a difference of one band with other isolates in PstI. This isolate (No. 3931) was considered similar to the other isolates of cluster 3. Indeed, differences of one band between isolates were observed previously 9 and are likely to result from epidemiologically irrelevant genetic changes.

\section{Antibiogram Typing}

Inhibition zone diameters of MRSA isolates are shown in the Table. Similarity between isolates using the inhibition zone diameters were computed with the Euclidean distance. For example, the Euclidean 


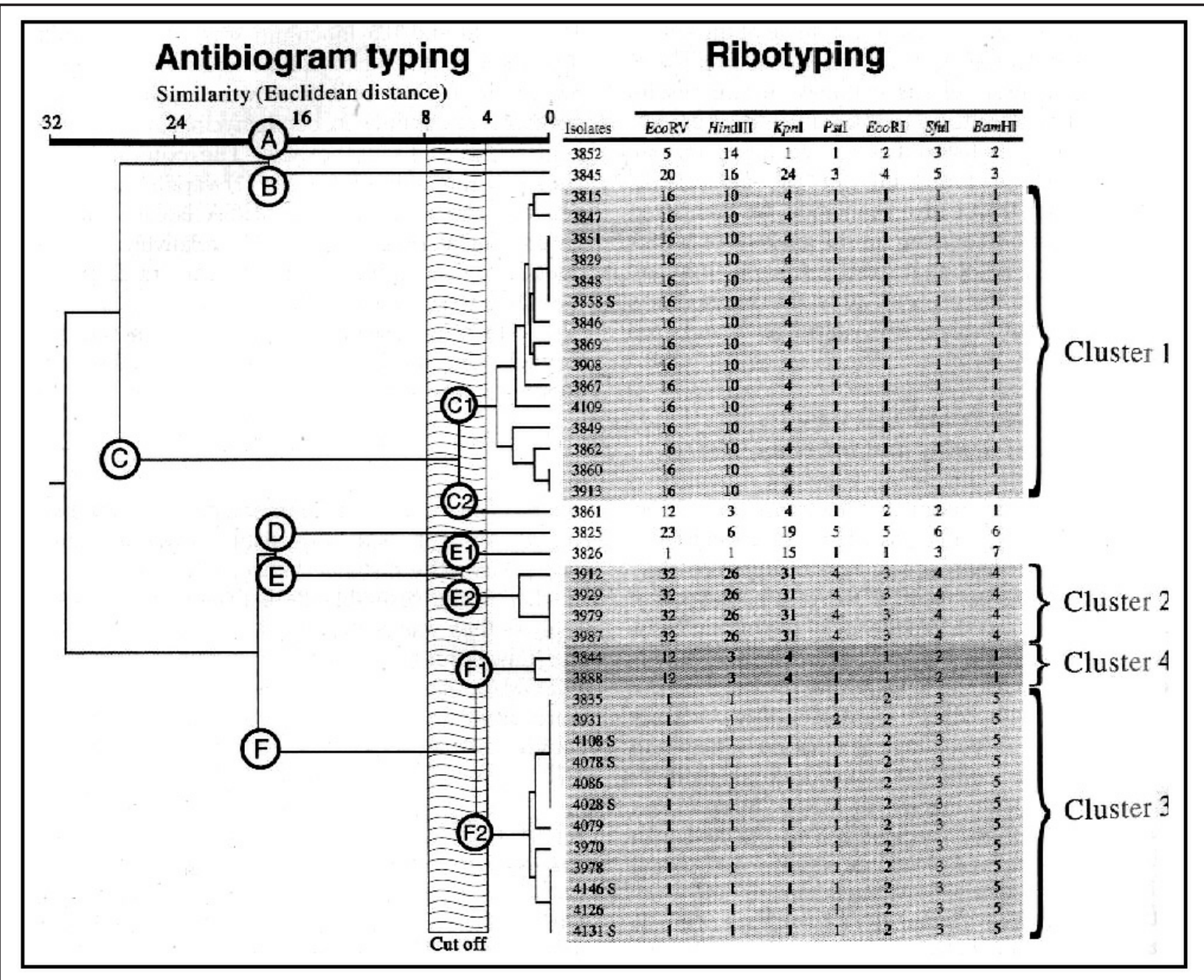

FIGURE 1. Dendrogram of similarities between methicillin-resistant Staphylococcus aureus isolates using antibiogram typing and comparison with ribotyping results. Ribotyping patterns obtained with each restriction enzyme are indicated by a number, and isolates were considered as having the same ribotype when sharing the same patterns for all enzymes (see text for discussion of isolate 3931). An "S" after the isolate's number indicates a staff member. Isolate 3848 was obtained from the spouse of one patient (isolate 3849).

distance between isolates No. 3852 and No. 3815 is:

$$
E_{3852-3815}=\sqrt{(10-28)^{2}+(25-27)^{2}+(23-21)^{2}+(7-7)^{2}+(33-7)^{2}}=31.7
$$

Such calculation was applied to all pairs of isolates. Cluster analysis (UPGMA) of these similarity data then was computed to produce a dendrogram (Figure 1).

A cut-off of the Euclidean distance may be defined as the value below which two isolates should be considered as similar. This cut-off previously was estimated as 8 , because it was found that the Euclidean distances derived from repeated antibiogram of 126 isolates were $<8$ in $98.4 \%$ of the cases. ${ }^{8}$ Thus, the dendrogram in Figure 1 shows the existence of three major groups of isolates $(\mathrm{C}, \mathrm{E}$, and $\mathrm{F}$ ) and three distinct isolates only remotely related to these groups (A, B, and D). By analyzing the structure of this dendrogram, major groups can be divided further (F1 and F2, E1 and E2, C1 and C2). Thus, nine distinct strains could be discriminated by antibiogram typing, among which five comprised only one isolate, and four comprised multiple isolates. These results are in concordance with the results of ribotyping (Figure 1). Therefore, in the present study, the value of the cut-off could be set up between 4 and 8 , according to the structure analysis of the dendrogram and the ribotyping data.

\section{Epidemiological Analysis}

The four clusters identified by the typing methods then were compared to epidemiological data (ward and period of stay; Figure 2).

Cluster 1. The first isolate of cluster 1 was obtained in July 1992. From then on, this antibiotype was isolated in 17 patients (13 in 1993) from five different wards and from the spouse of one patient (No. 
3848, Figure 1). One nurse was found to be positive in the internal medicine ward in 1992 and another in the dermatology ward in 1993. Considering each ward separately, all patients but one (patient No. 13 of cluster 1 in Figure 2) were hospitalized during the same period and cared for by the same nursing team (6 patients in the orthopedic ward, 5 patients in the dermatology ward, 3 patients in the internal medicine ward, and 2 patients in the septic surgery ward). However, no clear explanation could be found as to how this strain had spread from one ward to another during the whole period (1.5 years). Transmission by staff members is a possibility, but only two (internal medicine and dermatology) of 303 staff members were found to be transient nose carriers of the strain upon screening. Moreover, staff normally are dedicated to one ward only, except for some of the medical staff who serve both the orthopedic and the septic surgery wards.

\section{Clusters 2 and 3}

All except one isolate (patient No. 2 of cluster 2 in Figure 2) of clusters 2 and 3 were found in one ward (septic surgery). Screening for MRSA in the hospital staff, in November 1993, revealed that five members were colonized with isolates similar to the isolates of the patients of cluster 3 . The first isolate of this cluster was identified in May 1993 from a patient who remained colonized until his discharge from the hospital in August. This carriage of MRSA during a long-term hospitalization (4 months) may have been responsible for the spread of this strain to the hospital staff and to other patients, despite control measures. ${ }^{10}$ Moreover, the patient probably acquired the strain in 1990, when he was hospitalized in a ward that had an outbreak with a strain showing the same ribotype and antibiogram.

\section{Cluster 4}

Both patients of cluster 4 were hospitalized in the same ward (neurosurgery) at a 10-day interval.

\section{DISCUSSION}

The majority of the typing procedures performed today deal with isolates suspected to be the cause of an ongoing, or perhaps even terminated, epidemic. Real-time typing of clinical isolates able to cause epidemics could be a tool for the early detection of an increased occurrence of a single strain and thereby might allow early intervention and prevention of epidemics. However, most of the modern molecular typing methods are costly, technically demanding, and not feasible in small laboratories. Antibiogram typing

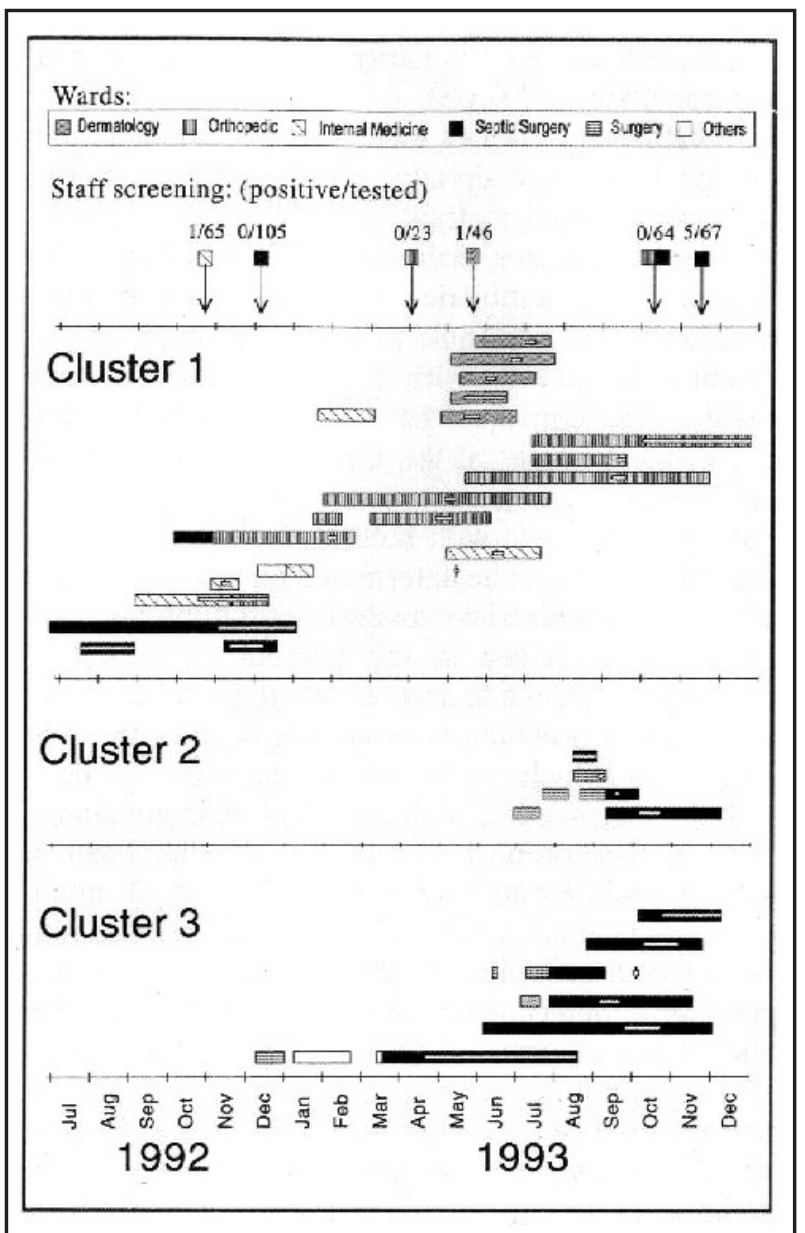

FIGURE 2. Hospitalization of case-patients of clusters 1,2 , and 3 , according to wards (one line per patient). The rectangles represent the hospitalization duration, and the internal white bars indicate the periods during which the patient was known to be positive for methicillinresistant Staphylococcus aureus (MRSA; interrupted rectangles indicate discharge and readmission). Arrows indicate surveillance cultures in the staff members (number of positive/number investigated), and the squares at the beginning of the arrows indicate the investigated ward. Screening for MRSA among the hospital staff was performed on seven occasions: internal medicine ward, October 1992; septic surgery ward, December 1992, October and November 1993; orthopedic ward, April and October 1993; dermatology ward, June 1993.

using inhibition zone diameters may allow such realtime typing, as it can be performed readily in 1 day. In the present study, this method appeared to be as accurate as ribotyping in detecting MRSA clusters in our hospital, confirming previous results. ${ }^{8}$ Carriers of the same strains among hospital staff also were identified correctly. Moreover, the occurrence of two different clusters (2 and 3, Figure 2) in the same ward at the same time could be discriminated. Thus, antibiogram typing may provide useful data for the early detection and investigation of an outbreak of MRSA in a hospital. The prerequisites for the use of this methodology are the recording of quantitative data of the antibiograms (zone diameters) and the 
availability of software for similarity and cluster analysis (approximately \$200 US).

Other approaches for the use of antibiograms as a typing method already have been proposed. The more widespread method is the resistance profile, which characterizes each strain by its resistance to a defined set of antibiotics. By using 15 antibiotics, Struelens et al were able to get a high index of discrimination (0.959) with this method. ${ }^{12}$ Another method was proposed that takes into consideration the mean diameter of the inhibition zone using 11 antibiotics. ${ }^{13} \mathrm{~A}$ good discrimination also was obtained. However, with both of these methods, the reproducibility can be determined only arbitrarily. By using the multivariate analysis of inhibition zone diameters proposed in the present study, reproducibility is taken into account when the cut-off value is defined by preliminary experiments, and only a few antibiotics (which can be selected according to their stability) are needed to obtain a high discrimination.

Application of this method to another hospital setting requires an appropriate selection of antibiotics and estimation of the cut-off. Antibiotics should be selected according to the following criteria: (1) resistance determinants to the various antibiotics should be independent; (2) the isolates of the hospital should not be uniformly susceptible or resistant; and (3) the use of the test antibiotics in the hospital should be low, to minimize selective pressure. In addition, preference should be given to antibiotics for which resistance is coded on the chromosome and to antibiotics that have a well-defined border of the inhibition zone diameter. The optimal number of antibiotics that should be used was not estimated, but the five different drugs we used appeared to be adequate in the setting of our hospital. If additional or other antibiotics are used, a cut-off different from the value obtained in the present study might be expected.

It is important to note that the cut-off value must be defined according to each laboratory and hospital setting. This value first should be estimated by repeated testing of a large number of isolates and set up in order for $>95 \%$ of the distances between the first and the second test to be smaller than the cut-off. Then, using epidemiological data, a second estimation of the cut-off should be performed by analysis of the structure of the dendrogram. Because of the potential variation of this phenotypic method over time in a given setting, it should be reevaluated periodically. The method also might be validated once using a molecular typing method. Afterward, the latter method may be reserved for situations in which epidemiological and antibiotyping data are in conflict or are inconclusive.
TABLE

InHibition Zone Diameters of Methicillin-Resistant STAPHYLOCOCCUS AUREUS ISOLATES (IN MM)

\begin{tabular}{|c|c|c|c|c|c|}
\hline Isolates & $\mathbf{E}$ & CC & SXT & GM & CIP \\
\hline 3852 & 10 & 25 & 23 & 7 & 33 \\
\hline 3815 & 28 & 27 & 21 & 7 & 7 \\
\hline 3845 & 27 & 25 & 28 & 7 & 30 \\
\hline 3825 & 7 & 24 & 26 & 22 & 8 \\
\hline 3826 & 7 & 7 & 29 & 22 & 12 \\
\hline 3851 & 27 & 27 & 22 & 7 & 7 \\
\hline 3829 & 27 & 27 & 22 & 7 & 7 \\
\hline 3844 & 7 & 7 & 23 & 7 & 11 \\
\hline 3849 & 27 & 28 & 25 & 7 & 7 \\
\hline 3848 & 27 & 27 & 22 & 7 & 7 \\
\hline 3847 & 28 & 27 & 22 & 7 & 7 \\
\hline 3846 & 27 & 28 & 22 & 7 & 7 \\
\hline 3835 & 7 & 7 & 25 & 7 & 7 \\
\hline 3861 & 28 & 30 & 23 & 7 & 12 \\
\hline 3862 & 29 & 28 & 24 & 7 & 7 \\
\hline 3860 & 29 & 29 & 24 & 7 & 7 \\
\hline 3858 & 27 & 27 & 22 & 7 & 7 \\
\hline 3867 & 26 & 27 & 21 & 7 & 7 \\
\hline 3869 & 27 & 27 & 21 & 7 & 7 \\
\hline 3888 & 7 & 7 & 23 & 7 & 12 \\
\hline 3908 & 27 & 28 & 21 & 7 & 7 \\
\hline 3912 & 7 & 7 & 29 & 23 & 7 \\
\hline 3913 & 29 & 29 & 24 & 7 & 7 \\
\hline 3929 & 7 & 7 & 29 & 25 & 7 \\
\hline 3931 & 7 & 7 & 25 & 7 & 7 \\
\hline 3970 & 7 & 7 & 23 & 7 & 7 \\
\hline 3979 & 7 & 7 & 29 & 25 & 7 \\
\hline 3978 & 7 & 7 & 24 & 7 & 7 \\
\hline 3987 & 7 & 7 & 29 & 25 & 7 \\
\hline 4028 & 7 & 7 & 25 & 7 & 7 \\
\hline 4079 & 7 & 7 & 26 & 7 & 7 \\
\hline 4086 & 7 & 7 & 25 & 7 & 7 \\
\hline 4078 & 7 & 7 & 25 & 7 & 7 \\
\hline 4109 & 28 & 29 & 22 & 7 & 7 \\
\hline 4108 & 7 & 7 & 25 & 7 & 7 \\
\hline 4126 & 7 & 7 & 24 & 7 & 7 \\
\hline 4131 & 7 & 7 & 24 & 7 & 7 \\
\hline 4146 & 7 & 7 & 24 & 7 & 7 \\
\hline
\end{tabular}

Abbreviations: E, erythromycin; CC, clindamycin; SXT, cotrimoxazole; GM, gentamicin; CIP, ciprofloxacin.

In conclusion, antibiogram typing using inhibition zone diameters may be a powerful tool for the prospective surveillance and control of MRSA in some hospitals, provided that the strains of MRSA are not yet uniformly resistant to a certain number of antibiotics. The results of the present study showed a good agreement between antibiogram typing, ribotyping, and epidemiological analysis. The method is 
simple, rapid, and readily available and thus may be especially suitable for hospital laboratories that do not have sophisticated typing facilities. It allows the early detection of epidemics and thus permits the concentration of infection control efforts.

\section{REFERENCES}

1. Blumberg HM, Rimland D, Kiehlbauch JA, Terry PM, Wachsmuth IK. Epidemiologic typing of Staphylococcus aureus by DNA restriction fragment length polymorphisms of rRNA genes: elucidation of the clonal nature of a group of bacteriophage-nontypeable, ciprofloxacin-resistant, methicillin-susceptible $S$ aureus isolates. J Clin Microbiol 1992;30:362-369.

2. Costas M, Cookson BD, Talsania HG, Owen RJ. Numerical analysis of electrophoretic protein patterns of methicillin-resistant strains of Staphylococcus aureus. J Clin Microbiol 1989;27:2574-2581.

3. Nicolle L, Bialkowska-Hobrzanska H, Romance L, Harry VS, Parker S. Clonal diversity of methicillin-resistant Staphylococcus aureus in an acute-care institution. Infect Control Hosp Epidemiol 1992;13:33-37.

4. Preheim L, Pitcher D, Owen R, Cookson B. Typing of methicillin resistant and susceptible Staphylococcus aureus strains by ribosomal RNA gene restriction patterns using a biotinylated probe. Eur J Clin Microbiol Infect Dis 1991;10:428-436.

5. Trilla A, Nettleman MD, Hollis RJ, Fredrickson M, Wenzel RP, Pfaller MA. Restriction endonuclease analysis of plasmid DNA from methicillin-resistant Staphylococcus aureus: clinical application over a three-year period. Infect Control Hosp Epidemiol 1993;14:29-35.
6. Wei MQ, Wang F, Grubb WB. Use of contour-clamped homogeneous electric field (CHEF) electrophoresis to type methicillin-resistant Staphylococcus aureus. J Med Microbiol 1992;36:172-176.

7. Giacca M, Menzo S, Trojan S, Monti-Bragadin C. Cluster analysis of antibiotic susceptibility patterns of clinical isolates as a tool in nosocomial infection surveillance. Eur J Epidemiol 1987;3:155-163.

8. Blanc DS, Lugeon L, Wenger A, Siegrist HH, Francioli P. Quantitative antibiogram typing using inhibition zone diameters compared with ribotyping for epidemiological typing of methicillin-resistant Staphylococcus aureus. I Clin Microbiol 1994;32:2505-2509.

9. Lugeon C, Blanc DS, Wenger A, Francioli P. Molecular epidemiology of methicillin-resistant Staphylococcus aureus at a low incidence hospital over a 4-year period. Infect Control Hosp Epidemiol 1995;16:260-267.

10. National Committee for Clinical Laboratory Standards. Performance standards for antimicrobial susceptibility testing. M2-A4. Villanova, PA: NCCLS; 1991.

11. Blanc DS, Siegrist HH, Sahli R, Francioli P. Ribotyping of Pseudomonas aeruginosa: discriminatory power and usefulness as a tool for epidemiological studies. J Clin Microbiol 1993;31:71-77.

12. Struelens MJ, Deplano A, Godard C, Maes N, Serruys E. Epidemiological typing and delineation of genetic relatedness of methicillin-resistant Staphylococcus aureus by macrorestriction analysis of genomic DNA by using pulsed-field electrophoresis. J Clin Microbiol 1992;30:2599-2605.

13. Tenover FC, Arbeit R, Archer G, et al. Comparison of traditional and molecular methods of typing isolates of Staphylococcus aureus. J Clin Microbiol 1994;32:407-415.

\section{Drug-Resistant Acinetobacter baumanii}

\section{Gina Pugliese, RN, MS} Martin S. Favero, PhD

Acinetobacter baumanii has been reported increasingly as a nosocomial pathogen worldwide, especially in outbreaks occurring in intensive-care units. This organism is a typical gramnegative water bacterium that has the fascinating ability to grow in all types of water and then to be distributed to environmental surfaces, some of which may become part of a colonization or infection route.

Dr. Xavier Corbella and coinvestigators from the University of Barcelona, Spain, evaluated fecal colonization with multidrug-resistant A baumanii in 189 consecutive patients admitted to three 12 -bed hos- pital intensive-care units (ICUs) during two different 2-month periods (October to November 1993 and May to June 1994). Rectal swabs were obtained from ICU patients on admission and weekly until discharge from the ICU. Overall, 77 (41\%) of 189 patients were found to be colonized with multidrug-resistant $A$ baumanii during the first week; 10 (13\%) during the second week; 9 (12\%) during the third week; and 3 (4\%) during the fourth to fifth weeks. Rectal swabs taken from 20 patients within the first 48 hours already were positive; 8 of these patients were admitted to the hospital from the community and did not have previous hospitalization, and 12 were from other wards of the hospital. Multidrug-resistant $A$ baumanii was isolated from 5 of 26 environmental samples (3 monitor touch pads, 1 ventilator tube, and 1 floor sample), as well as from 3 of 5 latex gloves worn by staff members who had been touching patients.

The authors conclude that the digestive tract in severely ill patients can be a reservoir for $A$ baumanii. In addition, the authors believe that the positive cultures of latex gloves worn by staff supports the relevance of cross-transmission of $A$ baumanii via gloved hands of staff.

FROM: Corbella X, Pujol M, Ayatts J, et al. Relevance of digestive tract colonization in the epidemiology of nosocomial infections due to multiresistant Acinetobacter baumanii. Clin Infect Dis 1996;23:329-334. 